\title{
EL LIBRO DE FAMILIA DE LOS AYLLÓN-MAYORGAS (MEDINA SIDONIA, SIGLOS XVI-XVIII)
}

\author{
Belén Piqueras García \\ Arturo Morgado García \\ UNIVERSIDAD DE CÁDIZ
}

\section{RESUMEN}

Los libros de familia fueron uno de los géneros predilectos de lo que Amelang llama Autobiografía popular. En este artículo realizamos el análisis de los rasgos externos y del contenido de un libro de familia de la Medina Sidonia de los siglos XVI a XVIII. Nos proporciona información acerca de las fechas de nacimiento, matrimonio y defunción de los componentes del clan Ayllón-Mayorgas, así como muchos datos de fundaciones piadosas realizadas en la Medina de los siglos XVII y XVIII, junto con algunas observaciones personales de interés.

Palabras clave: Medina Sidonia (Cádiz, España), siglos XVI a XVIII, cultura escrita, libro de familia, iglesia.

\section{ABSTRACT}

Family books were one of the predilect genres of Popular Autobiography, using the terminology created by James Amelang. In this article we analize the external aspects and the content of a family's book of Medina Sidonia of the XVIth, XVIIIth and XVIIIIth centurias.It gives information about the dates of birth, wedding and death of the members of the family AyllonMayorgas, so a lot of informaion about pious foundations realizated in Medina Sidonia during XVIth, XVIIth and XVIIIth centuries, so some personal observations which have big interest.

Key words: Medina Sidonia (Cádiz, Spain), XvIth, XVIIth, XVIIIth centuries, history of the writing culture, family's book, Church.

La escritura popular autobiográfica adopta numerosas formas a lo largo del Antiguo Régimen, ya sean autobiografías, memorias, diarios, autobiografías espirituales, relatos de viajes, o libros de familia. Estos últimos han sido definidos como formas de escritura plurales 
parecidas a los diarios cuyo objeto (y sujeto) principal es la familia a cuyo complejo aparato memorístico contribuyen ${ }^{1}$. Aunque compartían con los diarios la misma estructura de anotaciones discontinuas, los libri di ricordanze, livres de famille, Hauschkroniken y otros textos de la misma índole, se distinguen de los diarios por la falta de referencias a la experiencia individual, siendo los temas principales el tiempo, las cosechas, los precios, los desastres y sucesos naturales, las enfermedades, los delitos y sus castigos, así como otras cuestiones más ligadas al patrimonio, registrando mucha información de interés y uso familiar ${ }^{2}$. La voluntad de crear memoria es evidente, surgiendo los primeros en la Toscana del siglo XIV ${ }^{3}$, y difundiéndose a otras zonas del continente europeo durante la centuria posterior ${ }^{4}$.

Presentamos en esta ocasión el análisis de un "libro", siguiendo la calificación con la que este cuadernillo inicia su primera página "Libro donde se allaran escriptas las descendencias de Albaro de Ayllón y de María de Medina, mis bisabuelos, por parte de madre". Se conserva en la biblioteca del Seminario de San Bartolomé de Cádiz, guardado junto a otros documentos en una carpeta sin seguir ningún criterio de clasificación, dado que es en el momento actual cuando se ha iniciado la tarea de organización de dicha biblioteca.

Su estado de conservación es regular, está realizado en papel tamaño cuartilla (excepto una hoja central que tiene el tamaño de folio, aunque aparece plegada guardando las dimensiones del conjunto escrito) y lo conforman cuarenta hojas, sin foliar, unidas en su margen izquierdo por hilo bramante.

El análisis de su contenido, así como el tipo de escritura utilizado lleva a dividirlo en dos partes, ejecutadas cada una de ellas en distinta época y por diferente persona. La primera abarcaría las doce primeras hojas, en las cuales se refiere el linaje de la familia Mayorgas-Ayllón, remontándose en su exposición a principios del siglo XVI y abarcando hasta la segunda mitad del siglo XVII, debiendo ser escrita a finales del siglo XVII o inicios del XVIII por un hijo de Francisco de Mayorgas y de Beatriz de Ayllón, casados en 1639, pero no podemos asegurar por cual de sus trece hijos ya que no puede deducirse del contenido.

La segunda parte, integrada por las veinte y ocho hojas finales, la dedica a referir "las capellanías, memorias y patronatos de los derechos que me corresponden por la parte de mi madre, las que referiré en la forma siguiente", según se lee en su inicio. La primera hoja de esta parte es de tamaño folio, plegada a la mitad, en la que a modo de cuadro gráfico (foto 1) se intercala un árbol familiar. Esta parte sería realizada a finales del siglo XVIII por Alonso Montes de Oca Hurtado y Novela, hijo de Juan Montes de Oca Villacreces y Francisca Hurtado y Novela, casados en 1720, su autor formal, y casi con toda seguridad su autor material, y a quien podemos suponer el autor formal del cuadernillo en su integridad ya que sería quien lo idease, anexando la primera parte, cuyo autor sería un antepasado suyo. Nuestro hombre sería bautizado en Medina Sidonia el 27 de julio de 1727 y contraería matrimonio con María Garcés y Villavicencio,

1 MORDENTI, R., "Su alcuni problemi di metodología della recerca. Per un archivio della scritura popolare: materiali di lavoro", Revista di Studi Storici, 1-2, 1987.

2 AMELANG, James S., El vuelo de Icaro. La autobiografía popular en la Europa Moderna, Madrid, Siglo Xxi, 2003, pp. 22-23. Más concretamente, MORDENTI, R., "I libri di famiglia in Italia, II. Geografia e Storia", en Apéndice gli Atti del Seminario nazionale su I libri di famiglia in Italia: quindici anni di ricerche, Roma, Edizione di Stortia e Letteratura, 2001.

3 Aunque también los hay con la misma antigüedad en Francia: vid. DELMAIRE, "Le livre de familla des Le Borgue (Arras 1347-1538)", RN, 65, 1983. Otro ejemplo galo, en DARE, Nicolas, "Memoires et livre de familla de Nicolas Dare", Collection des documents inédits relatifs á la ville de Troyes et a la Champagne publiés par la Société ácademique de l'Aube, vol. III, Troyes, 1886.

4 CASTILLO GOMEZ, Antonio, "Entre la necesidad y el placer. La formación de una nueva sociedad del escrito (siglos XII-XV)", Historia de la cultura escrita, Gijón, Ediciones Trea, 2001, p. 200. Numerosas referencias concretas, aunque sin citar expresamente los libros de familia, en SIMON TARRES, Antoni, "Memorias y diarios personales de la Cataluña Moderna”, Historia social, 2, 1988. Más reciente (2000) es la obra de X. Torras sobre los libros de familia payeses. 
natural de Vejer, el 1 de mayo de 1770, en la Iglesia mayor de Vejer, celebrando el matrimonio su tío, el padre comendador Fray Francisco de Santa Rosalía. De dicha unión nacerían Juan José Francisco de Paula (1771), Tomasa (1772), Francisco (1775), Joseph María del Carmen (1777), y Juan José Calixto (1779), especificándose de algunos incluso la fecha de confirmación.

Aunque los autores, y el uso de la memoria escrita nos permite llegar a esta conclusión, se mueven en una esfera relativamente instruida y culta que contrasta con los elevados niveles de analfabetismo existentes en su localidad ${ }^{5}$, se aprecia entre ellos, empero, un grado distinto en el empleo de una cuidada gramática, estando más cercano a ella el primero que el segundo, en cuyo texto constatamos mayores indecisiones gráficas (se utiliza indistintamente $\mathrm{s} / \mathrm{c} / \mathrm{z}, \mathrm{b} / \mathrm{v}$ o $\mathrm{h} / \mathrm{f}$ ), siendo corriente escribir la misma palabra de distintas formas (a/ha, avito/habito, ella/hella, habuelos/abuelos/avuelos, ijo/hijo), constatándose que el autor no sigue las normas gramaticales en su ejecución, situación de la que no escaparía gran parte de la población alfabetizada del momento, a pesar de los intentos realizados en los siglos XVII y XVIII por fijar dichas normas.

Con independencia de la doble división indicada, el objetivo perseguido a la hora de conformar este testimonio escrito es único para todo el conjunto, perpetuar en la memoria unos datos de interés. Alonso Montes de Oca Hurtado y Novela, consciente del poder de la escritura como medio para salvaguardar la memoria de la familia y los derechos que le correspondían, idearía la confección de este cuaderno en su integridad para dejar constancia escrita que garantizase

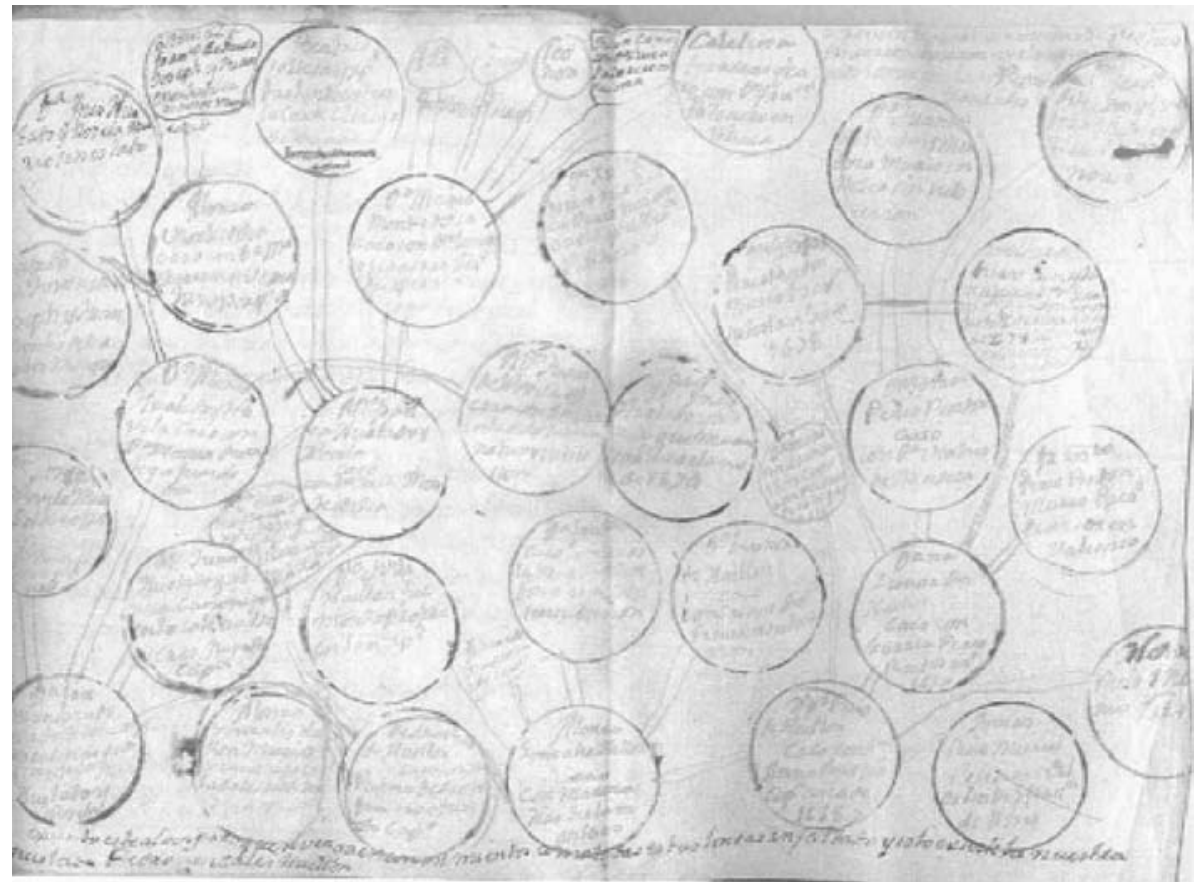

Foto 1: Árbol genealógico de la familia Mayorgas-Ayllón.

$5 \quad$ PASCUA SANCHEZ, María José de la, "Aproximación a los niveles de alfabetización en la provincia de Cádiz (las poblaciones de Cádiz, El Puerto de Santa María, Medina Sidonia y Alcalá de los Gazules entre 1675 y 1800)”, Trocadero, 1, 1989. 
su perpetuidad. Al realizar este cuaderno sus autores no pretenden una constancia documental como fe pública de lo contenido, no se confecciona un documento y por tanto no se incorporan ninguno de los requisitos exigidos para que se demuestre su validez. No detalla el nombre de su autor, ni de su destinatario, ni se incluyen cláusulas ni elementos validatorios, sino que de una forma subjetiva se expone la memoria de la familia, constituyendo un ejemplo de "escritura del yo". Plasman los datos como recuerdo, y para que tengan una base de credibilidad van remitiendo a los documentos que pueden dársela, libros de bautismo, de matrimonio o testamentos, lo que a su vez nos indica que sus autores no sólo no eran analfabetos, sino que además conocían la importancia de la documentación como base o apoyatura legal de todo lo que plasman y anexan en el cuaderno, aludiendo con ello indirecta pero claramente a su relación con la lectura. Todo ello, unido a la forma de estructurar el contenido, en el que se intercalan cuadros con el ánimo de ofrecer un discurso que resulte más claro a quien lo consulte, la inclusión de citas en latín, e incluso la forma de concluirlo, con la inserción de una coplilla, nos lleva a concluir que eran personas familiarizadas con la documentación y conocedoras de su importancia, de hecho, Alonso Montes de Oca tendría un archivo familiar, según se desprende de su narración "éste mi archivo".

\section{FACTURA EXTERNA DEL CUADERNO}

El cuadernillo es tamaño cuartilla, $16 \times 21$ centímetros, excepto la hoja inicial de la segunda parte, que tiene el tamaño de folio, 28x21 centímetros, aunque aparece plegada guardando las dimensiones del conjunto escrito, conformando un total de cuarenta hojas escritas por ambos lados, recto y vuelto, sin foliar, y unidas en su margen izquierdo por un hilo bramante cosido a las mismas.

El material utilizado como soporte gráfico es el papel, que presenta un tono amarillento y en el que además de poder apreciar las marcas de puntizones y corondeles, también en algunas de sus hojas son perceptibles marcas de agua o filigranas correspondientes a diversas familias: buey, caballo, círculo, estrella, no faltando algunas catalogadas como indeterminadas y otras referidas a apellidos (Costa y Motaro). Debemos precisar que en la segunda parte se utiliza papel de diversa calidad: uno de textura más fina, de aspecto más cuidado y de color menos amarillento, coincidente con el que incluye en su filigrana el apellido Costa, y un segundo tipo de textura más rugosa.

El color de la tinta es ocre, aunque su tonalidad en algunas de las hojas es tan clara, sobre todo en la segunda parte, que llega a resultar casi imperceptible, lo cual hace casi imposible su lectura.

La escritura utilizada responde en general, a pesar de que sus dos partes fueron escritas en diferente fecha y por distinta mano, al tipo que vendría derivado de la escritura humanística inclinada y que ocupará el panorama gráfico desde finales del siglo XVI hasta finales del XVII, caracterizado por el gusto manierístico en consonancia con la estética barroca imperante ${ }^{6}$, siendo este cuaderno un claro ejemplo del multigrafismo ${ }^{7}$ propio de la época.

6 Esta definición es la que da Elisa Ruiz en su estudio sobre "la escritura humanística y los tipos gráficos derivados", en RIESCO TERRERO. Angel (ed.), Introducción a la Paleografía y la Diplomática General, Madrid, Síntesis, 1999, pp. 149-176.

7 Este multigrafismo era habitual en la élite instruida: de hecho, en 1754 se exigía a los maestros gaditanos de primeras letras "saber formar con propiedad la letra bastarda literal y detenida, la grifa, romanilla y redonda, y demás que se usaren; dando razón de la simetría composición y arte de dichas letras de doce, diez y seis y demás tamaños, escribiendo práctico de todos ellos" (MORGADO GARCIA, Arturo, El clero gaditano a fines del Antiguo Régimen. Estudio de las órdenes sacerdotales (1700-1834), Cádiz, Fundación Municipal de Cultura, 1989, p. 65). 
Dicha escritura está dispuesta paralela al borde estrecho de la hoja, no siguiendo con rigor el guardar los márgenes en ninguna de las partes. Y en los casos en que aparece espacio marginal lo suele emplear para incorporar anotaciones, algunas de las cuales se aprecia que fueron incorporadas con posterioridad al resto del escrito, e incluso en ocasiones nos aventuramos a decir que por una mano distinta. El texto se dispone a línea tendida, excepto en las hojas en las que su autor intenta una ordenación del mismo a modo de columnas, persiguiendo una finalidad aclaratoria del contenido de su escrito. A todo lo dicho escapa la primera hoja de la segunda parte, ya que en ella la escritura aparece paralela al borde ancho de la hoja y está trazada dentro de círculos, ya que la intención de su autor es realizar un árbol genealógico.

A pesar de que en la confección escrita del documento intervienen distintas manos, en su conjunto responde, como hemos indicado, al tipo gráfico derivado de la llamada humanística inclinada, pudiendo hablar de caracteres comunes a todo el documento, como son escritura minúscula, ductus mayoritariamente uniforme (siendo menor la uniformidad en la segunda parte, en la que alternan hojas de una gran perfección gráfica con otras realizadas con mayor descuido y cursividad, como por ejemplo la 36), grado de inclinación de los astiles a la derecha, formas regulares y con clara separación entre las palabras y escasísimas correcciones (en su lugar se encuentran inserciones, realizadas con posterioridad, con el ánimo de aclarar o completar lo ya escrito), escaso empleo de abreviaturas que, cuando aparecen, suelen ser de contracción y en las que se utiliza el sistema de abreviar por letras sobrepuestas, y, en general, inexistente uso de sistema de puntuación (aunque en la primera parte sí intenta, en algunas ocasiones, marcar el inicio de algunos párrafos, así como el final, mediante dos pequeños trazos paralelos). En ambas partes se inserta como elemento figurado al inicio de cada hoja, centrado en su margen superior, un signo, que en las doce primeras hojas corresponde a dos líneas paralelas, realizadas con inclinación a la izquierda y sobrepuesto a ellas un trazo sinuoso similar a la letra " $r$ ", y en la segunda parte observamos, no en todas sus hojas pero sí en la mayoría, en la misma posición y lugar antes indicados, la cruz cursiva.

A pesar de todas las similitudes, resaltan más las diferencias, siendo obvio que la primera parte está más próxima al molde de la escritura bastarda, en cuanto que en ella pueden apreciarse, además de las características indicadas, los siguientes rasgos: los caídos de la y griega, la h y la $\mathrm{p}$ tienden, tras haber llegado al límite inferior, a subir de forma paralela hasta enlazar con el signo siguiente, suele emplear el punto sobre la i latina, cuya forma oscila entre el característico punto o bien un trazo a modo de acento y tiende a unir letras dentro de una misma palabra. Si la primera escritura, en definitiva, es de módulo más pequeño y de trazado más anguloso e inclinado, la segunda presenta un mayor módulo y menor inclinación a la derecha, apreciándose no sólo separación entre las palabras, sino entre las letras de una misma palabra.

\section{ANÁLISIS DEL CONTENIDO.}

El tenor de este testimonio escrito en su conjunto persigue el mismo fin: dejar constancia escrita de una línea familiar, para de este modo garantizar los derechos que les correspondían sobre el patronato de ciertas capellanías.

En las doce primeras hojas el autor va detallando su línea familiar, tanto paterna como materna, "Libro donde se allaran escriptas las descendencias de Albaro de Ayllón y de María de Medina, mis bisabuelos por parte de madre”, remontándose para ello hasta la cuarta generación ("y así mismo rason de mis terseros abuelos"), además, para completar la relación, indica "y de los parentes los trasbersales asta cuarto grado". La fecha más antigua que aparece aludida es la de 1503, llegando en su relación hasta el año 1692, casi dos siglos de relación de la línea familiar. El discurso se completa no sólo con la alusión al parentesco, lugar de origen o vecindad 
de sus parientes y a su estado y profesión, sino además con la alusión a fechas de bautismo (especificando, en gran número de ocasiones, el libro y el número de folio del mismo, en que se asienta dicho bautismo), matrimonio, testamento (indicando el nombre del escribano ante quien fue realizado) y defunción, lo que no solamente refleja su preocupación por documentar fielmente los datos que refiere, sino que además es un claro indicio de su relación no sólo con la escritura, sino también con la lectura.

Como lugares indicados donde nacieron o residieron los antepasados de los autores, se citan diferentes localidades, la mayoría correspondientes a la provincia de Cádiz, a su diócesis, y a algunas de Sevilla, no faltando alusiones a lugares de otras regiones, como Alagunilla (entonces en el obispado de Coria, hoy Lagunilla, provincia de Salamanca), Alcalá de los Gazules, Arcos de la Frontera, Cádiz, corral de Almaguer (cerca de Madrid), Chiclana, Espera, Granada, Jerez de la Frontera, Lebrija, Medina Sidonia (de donde procede el mayor número), Puerto Real, Puerto de Santa María, Sevilla, y Vejer (segundo lugar en importancia), aunque, evidentemente, predomina la vinculación a la urbe asidonense, reflejo de la profunda endogamia de los núcleos rurales ${ }^{8}$. En diversas ocasiones se alude a que algunos de ellos pasaron a Indias, tratándose siempre de varones ${ }^{9}$.

Los autores pertenecen a una clase social destacada, formando parte de una línea familiar en la que muchos de sus miembros eran eclesiásticos y escribanos públicos, no faltando alguna que otra alusión a cargos tales como abogado de los Reales Consejos, alcalde de los hijosdalgos, alférez mayor, canónigo, capitán, gobernador, licenciado, presbítero, recaudador, regidor, sargento mayor, e incluso se habla de un catedrático de decretales mayores de la Universidad de Sevilla, lo que nos muestra hacia dónde va encaminada la estrategia familiar, vinculada al mundo del funcionariado y la administración pública, y que destinará a muchos de sus hijos (es bastante frecuente tener tres o cuatro) a la carrera eclesiástica. Era algo habitual en la España del momento, siendo un ejemplo de esta "vocación clerical" el de los regidores madrileños, que en el siglo XVII destinan a una cuarta parte de sus descendientes a la Iglesia, si bien la proporción decaerá con fuerza en el Siglo de las Luces ${ }^{10}$. O por una serie de 42 familias cacereñas, todas ellas, a excepción de una, ofreciendo a sus hijos a la Iglesia como clérigos o monjas, en número de 105 y 248 respectivamente ${ }^{11}$. Ello no impediría, sin embargo, que algunos miembros de la familia tuvieran relaciones extramatrimoniales, aludiéndose en algunos casos, con total claridad, a hijos naturales: de Diego de Ayllón se menciona cómo "por ser público disen que fue su hijo Gaspar de Ayllón", naturalidad que contrastaría con el fuerte estigma social que conllevaría en el siglo XVIII la ilegitimidad, cuyo destino natural sería, directamente, la exposición ${ }^{12}$.

Algunos miembros del clan fueron incluso recordados por los cronistas de la ciudad: Alonso de Novela, por ejemplo, fue maestrescuela de la catedral de Cádiz a mediados del siglo XVII, tras haber sido vicario de la localidad asidonense. Francisco de Segura Ayllón, por su parte, fue deán del cabildo gaditano a inicios de dicha centuria, formando parte del cabildo colegial de Jerez de

8 Lo que se explica dado la escasa inmigración en los mismos, vid. BUSTOS RODRIGUEZ, Manuel, et al., "La población de la provincia de Cádiz en los siglos XVII y XVIII", Trocadero, 2, 1990.

9 Sobre las repercusiones sociales de dicha emigración masculina, PASCUA SANCHEZ, María José de la, Mujeres solas, Cádiz, Universidad, 1998.

10 HERNANDEZ, Mauro, A la sombra de la Corona. Poder local y oligarquía urbana, Madrid 1696-1808, Madrid, Siglo XXI, 1993.

11 RODRIGUEZ SANCHEZ, Angel, "El poder y la familia. Formas de control y de consanguinidad en la Extremadura de los tiempos modernos", CHACON JIMENEZ, Francisco, y HERNANDEZ FRANCO, Juan (eds.), Poder, familia y consanguinidad en la España del Antiguo Régimen, Barcelona, Anthropos, 1993.

12 Lo que se refleja en el hecho de que, con el tiempo, las distintas tipologías ilegítimas aparecidas en los documentos de bautismo van desapareciendo poco a poco, predominando de una forma nítida la de expósito. BUSTOS RODRIGUEZ, Manuel, et al., op. cit. 
la Frontera Juan de Novela y Alonso González Ayllón Maldonado ${ }^{13}$. Pedro de Mirabal Ayllón llegaría a ser obispo de Nápoles ${ }^{14}$. Fernando Montes de Oca y su hermano Luis, fueron respectivamente canónigos en Sevilla y Evora ${ }^{15}$. Alonso Novela de los Cameros, nacido en 1649, fue regidor de Medina Sidonia, alcalde por el estado noble de Jerez de la Frontera en 1716, y alférez mayor en Sanlúcar de Barrameda en $1724^{16}$. Cristóbal Montes de Oca se convertiría en oidor de la Chancillería de Granada en $1790^{17}$. Pedro de Novela Espínola, caballero del orden de San Juan, falleció combatiendo contra los ingleses en los mares del Ferrol a bordo del navío Princesa a mediados del siglo XVIII ${ }^{18}$. Juan Luis de Novela, oidor de la audiencia de Sevilla y antes de la de Valencia, sería autor de diferentes obras religiosas, como Esfuerzos del alma para ir a Dios. Camino del cielo y guía para la perfección (Sevilla, 1767), Conocimiento de sí propio en el tribunal de la conciencia: análisis del mundo (Sevilla, 1780) ${ }^{19}$.

Se detalla quienes fueron fundadores de capellanías, dónde las fundaron y en algunos casos a quienes nombraron o a quien correspondía el patronazgo, siendo precisamente el reconocimiento a este derecho el móvil desencadenante de la confección del cuaderno. Conviene recordar que en el siglo XVII se asiste en la diócesis de Cádiz a un auge fundacional de estas instituciones (en el caso concreto de Medina Sidonia, las 63 capellanías de 1605 se conviertieron en 321 en 1691 ${ }^{20}$, fenómeno también generalizado en el resto de España ${ }^{21}$, adquiriendo, sobre todo en el siglo XVIII, un fuerte carácter familiar, lo que se refleja en este cuaderno insistiendo continuamente en la pertenencia al linaje de los respectivos patronos. Aunque no contamos con estudios en profundidad para el caso de Medina Sidonia, sí sabemos que en el siglo XVIII, los eclesiásticos de esta localidad que se decantan por la fundación de una capellanía, eligen como capellanes a parientes, especialmente los sobrinos, decantándose también por el factor de la consanguineidad en la elección de patronos, siendo los sobrinos, por una parte, y las hermanas y su descendencia, por otra, preferidos a los elementos más significativos del clero asidonense, tales vicarios, curas u órdenes religiosas: la primacía del control familiar de las fundaciones sobre el eclesiástico es evidente ${ }^{22}$. Algo similar sucede en nuestra familia, donde los nombramientos de capellanes y patronos en el seno del propio linaje constituyen una moneda de cambio habitual, sin que en ningún momento se prefiera a la institución eclesiástica, lo que provocaría, de paso, la profunda endogamia geográfica del estamento eclesiástico: a inicios del siglo XVIII el $68 \%$ de los capellanes asidonenses habían nacido en dicha localidad ${ }^{23}$. No obstante, con el paso del tiempo, los derechos se van transmitiendo a otros linajes ante la extinción del clan fundador, como consecuencia, evidentemente, de matrimonios sin descendencia (bastante

\footnotetext{
13 MARTINEZ DELGADO, Francisco, Historia de la ciudad de Medina Sidonia, reed., Cádiz, Diputación Provincial, 1992, p. 291, pero redactada a finales del XVIII.

14 Ibidem, p. 305.

15 Ibidem, p. 306.

Ibidem, p. 315 .

Ibidem, p. 316

Ibidem, p. 317.

Ibidem, p. 324

20 MORGADO GARCIA, Arturo, El estamento eclesiástico y la vida espiritual en la diócesis de Cádiz en el siglo XVIII, Cádiz, Universidad, 1996, p. 40.

21 GONZALEZ RUIZ, Manuel, "Las capellanías españolas en su perspectiva histórica”, Revista Española de Derecho Canónico, V, 1950. Sobre el carácter patrimonial de estas fundaciones, PRO RUIZ, J., "Las capellanías: familia, iglesia y propiedad en el Antiguo Régimen”, Hispania Sacra, XLI, I, 1989.

22 MARTINEZ BAREA, Avelina, El bajo clero rural en el Antiguo Régimen (Medina Sidonia, siglo XVIII), Cádiz, Universidad, 2001, p. 155

23 MORGADO GARCIA, Arturo, "La vida espiritual en la diócesis de Cádiz a inicios del siglo XVIII", ARANDA DONCEL, J., Congreso de Religiosidad Popular en Andalucía, Córdoba, Cajasur, 1994.
} 
frecuentes, a tenor de lo indicado en el documento), solterías impenitentes o forzosas, emigraciones a Indias o un exceso de carreras eclesiásticas: de hecho, en la visita pastoral realizada a Medina Sidonia en 1717, no aparece ningún capellán apellidado Mayorgas o Ayllón, aunque sí un Alonso Hurtado de Novela, seglar natural de Medina Sidonia, y que detentaba las rentas de una memoria de misas ${ }^{24}$.

Las fundaciones no se limitan a las capellanías, sino que encontramos alusiones también a memorias de misas, la mayoría en la localidad de Medina Sidonia, pero también tenemos la fundada por Pedro González de Ayllón en la iglesia de San Pablo de los Reyes, en el reino del Perú, así como agregaciones de tierras y capital a los mayorazgos de la familia, y el patronato de obras pías fundado en 1634 por Juan Jiménez Barragán y su madre para dotar parientas pobres y huérfanas a fin de que pudieran elegir estado, siendo el patrono el deán y el cabildo catedralicio gaditano ${ }^{25}$. Sabemos también que en 1646 el vicario Alonso de Novela, en testamento otorgado ante el escribano Francisco Mayorga, fundó un patronato de obras pías cuya acción filantrópica beneficiaba a las viudas pobres de la localidad, a los curas y beneficiados por determinadas obligaciones litúrgicas y culturales, al convento de San Cristóbal, a algunas cofradías y a las huérfanas sin medios para contraer matrimonio ${ }^{26}$.

La intención prioritaria es, claramente, registrar los derechos del clan familiar: en esta línea, cabría destacar que el autor de la segunda parte indica que "soi patrono de las capellanías, memorias y patronatos de los derechos que me corresponden por la parte de mi madre, las que referiré en la forma siguiente", procediendo la línea a este derecho de Pedro González Ayllón, y el autor quiere dejar buena constancia de ello para que sus descendientes sepan el derecho que les corresponde: "le doi esta noticia a mis ijos para que sepan el derecho que les corresponde y que sirvan a Dios y a su María Santísima, para que nos lleve a la gloria, amén". El propósito del autor será el de perpetuar los derechos que le correspondían a capellanías, memorias y patronatos, (y para que nadie niegue este derecho, se hace alusión en numerosas ocasiones a los pleitos mantenidos por la familia para defender estos derechos) haciéndolo público y a la vez transmitiendo el poder que ello le confería. Poder que se extiende a otros elementos que tienden a magnificar la importancia del clan, como el derecho de sepultura y enterramiento en el interior de las iglesias: el autor, de este modo, nos relata cómo su padre fue enterrado en la Iglesia Mayor de Medina Sidonia "en la capilla del Sagrario de Varandillas, adentro sepulcro nuevo, donde nadie avia sido sepultado", y su madre fue enterrada igualmente en la Iglesia Mayor, "en la capilla de la Virgen de la Soledad".

El autor hace referencia también a las claúsulas testamentarias de alguno de los miembros de su familia, como su madre, Francisca Hurtado y Novela, fallecida en 1765 y enterrada en la Iglesia mayor, en la capilla de la Virgen de la Soledad, donando sus vestidos y alhajas a la virgen de la Paz y a cofradías pobres de la ciudad, favoreciendo con sus limosnas a muchos mendigos y sacerdotes enfermos, generosidad que no es de extrañar ya que "su vida fue mui exenplar", aunque esta acción caritativa fue frecuente en numerosos testadores asidonenses del siglo XVIII ${ }^{27}$.

Como nota a añadir, destacar el espíritu religioso que imbuye a Alonso Montes de Oca, lo que no es de extrañar dado el gran número de familiares eclesiásticos, y su carácter de patrón de numerosas capellanías, no resultando extrañas expresiones tales "que sirvan a Dios y

24 Archivo Diocesano de Cádiz, Manuscritos, libro 1238

25 Dicho patronato seguía existiendo en 1799, con unas rentas anuales de 19.282 reales (MORGADO GARCIA, Arturo, Iglesia y sociedad en el Cádiz del siglo XVIII, Cádiz, Universidad, 1989, p. 272).

26 Archivo Diocesano de Cádiz, Manuscritos, lib. 1238.

27 PASCUA SANCHEZ, María José de la, Vivir la muerte en el Cádiz del Setecientos (1675-1800), Cádiz, Fundación Municipal, de Cultura, 1991. 
a su $M^{a}$ Santísima, para que nos lleve a la gloria, amén" (referido el texto a sus hijos) o la inclusión en su escrito de la invocación monogramática, la típica cruz cursiva, o la referencia incluida al final, como colofón, y antes de escribir una coplilla del momento "los documentos que nos da el señor San Lucas en su santo evangelio cuando dize: que meritis putatis filius hominis veniet", concluyendo con la coplilla "de un siervo de Dios desengañado de la (borroso) desta miseria vida, dezía frecuentemente esta coplilla:
Amigos dos camas tengo,
Una en casa y otra en la yglesia.
La una viviendo me avriga,
La otra en muriendo me aguarda.
Que de la cama al sepulcro
Es muy corta la distancia.
Dios nos asista, amén”.

\section{UN EJEMPLO DE “ESCRITURA DEL YO”}

Este testimonio escrito evidencia ejemplarmente el uso que personas particulares, con independencia de su mayor o menor grado de alfabetización, hacían de la escritura ${ }^{28}$. A priori el cuaderno resulta interesante tanto por su contenido, que le confiere un gran valor para la historia local de la época, como por la pieza escrita en sí misma. La forma de referir de manera personal lo que se va escribiendo (movido por el deseo de dejar constancia escrita que ayudase a garantizar la perpetuidad de ciertos derechos) sin pretender evidentemente una constancia documental como fe pública de lo contenido, convierte a esta pieza escrita en un claro ejemplo de "escritura del yo". Plasma los datos como recuerdo vivo, persiguiendo dotarlos de veracidad al documentarlos, lo que a su vez nos indica, como ya hemos referido, que su autor conocía la importancia de la documentación como base para acreditar públicamente lo expuesto en el cuaderno, sospechando que su lectura podía resultar de interés e incluso ser fundamental, al menos para la descendencia familiar.

Los autores pertenecen a la sociedad alfabetizada, dominando la lectura y siendo conscientes de su necesidad, conociendo el poder que conllevaba el dominio de la lectura y la escritura. En el primer caso, Mayorgas-Ayllón, procedía por línea paterna de una familia de escribanos públicos, en concreto su padre y su abuelo ejercieron como tal en Medina Sidonia, de ahí que no sea de extrañar su destreza a la hora de escribir lo que él califica como "mi corto discurso", de forma clara, regular, dejando evidentes muestras del empleo de las normas gramaticales e inclusive introduciendo algunas fórmulas latinas, como la de "mutans mutandi", o dejando evidentes muestras de su familiaridad con la documentación "no e podido por ystrumento testificar".

En el segundo caso, constatamos igualmente el dominio de la escritura, aunque realizada de forma no tan reglada como en la primera parte, de hecho, y como ya hemos expuesto, se evidencia la ausencia de criterio en el uso de las normas gramaticales, siendo abundantes las indecisiones gráficas y ofreciendo poca uniformidad. No obstante, la escritura en su conjunto es clara, y muestra su pericia presentando una ordenación secuencial de la misma. No sólo inserta en la primera hoja el árbol genealógico, sino que para dar mayor claridad a lo que va refiriendo, dispone el texto en unos cuadros, especie de tabla esquemática del contenido. Y, como

28 Vid. CAFFARENA, Fabio, "Luchar con la escritura. Testimonio de "gente común” en el Archivo Ligure della scritura popolare (A.L.S.P.) de Génova”, Signo. Revista de la Historia de la Cultura escrita, 10, 2002. 
en el primer caso, el autor alude a documentación que debía consultarse para refrendar lo dicho por él, manifestándolo de esta forma "verlos llegando el caso" o "deste hallarán entre mis papeles". Otro ejemplo de la familiaridad del autor con lo escrito aparece referido cuando habla de su archivo: "se hallará entre mis papeles", "ver los papeles que todos están en el archivo", "este mi archivo". Y, como en el caso anterior, tampoco falta la inclusión de alguna que otra cita en latín: "purati qui meritis...putatis filius hominis veniet" (sic).

Los autores, especialmente el segundo, Alonso Montes de Oca, que es quien conforma el cuadernillo tal y como ha llegado hasta este momento, al realizarlo manifiestan implícitamente su voluntad de crear memoria para preservar la de sus antepasados, como garantía de la continuidad de los derechos que emanaban de la línea familiar, formada por personas que gozaban de ciertos privilegios, por lo que para que no se pierdan y permanezcan en la memoria, utilizan la escritura como instrumento recordatorio. Como dijera en 1560 Pedro de Navarra, en sus Diálogos de la diferencia del hablar al escrevir, al justificar las ventajas de lo último, porque "la palabra no dura más de cuanto es pronunciada, pero la escritura todo el tiempo que fuere conservada; y la palabra, si se oye, no se vee, pero la escritura se vee escrita y se oye, si es leyda, e la palabra no se comprende sino de cerca, pero la escritura se hace sentir en cabo del mundo"29. Se fosiliza en el espacio y en el tiempo, en forma de huella, como la fotografía, la realidad a la que se remite ${ }^{30}$.

NAVARRA, Pedro de, Diálogos de la diferencia del hablar al escrevir, Bellaterra, Setelle dell' Orsa, 1985, pp. 87-88. VIÑAO FRAGO, Antonio, "Por una historia de la cultura escrita: observaciones y reflexiones", Signo. Revista de Historia de la Cultura escrita, 3, 1996, p. 53, Universidad de Alcalá de Henares. 